The session on nuclear magnetic resonance was introduced by Prof. C. J. Gorter (Leyden), who reported on current work in this field in his laboratory. N. J. Poulis and G. E. G. Hardemann (Leyden) reported further measurements on a single crystal of cupric chloride. A. Lösche (Leipzig) reported on the use of nuclear resonance in studying the rate of slow polymerization by comparing the signal from protons in the solid (polymer) and liquid (monomer) phases. R. Extermann (Geneva) gave a review of the work of the Geneva group which has been particularly concerned with magnetic resonance at low fields. A. Abragam and W. Proctor (Saclay) discussed their work on 'spin calorimetry', one result of which is a trick for polarizing nuclei which have an inconveniently long spin-lattice relaxation time (weeks, say) in a matter of seconds. A. Abragam and I. Solomon (Saclay) have studied the spin echoes obtained when a nuclear absorption line is subject to quadrupole splitting. The results differ considerably from the usual ones, in that 'echoes' may be observed at $\tau / 2$ and $3 \tau / 2$ as well as $\tau$ after the stimulation. Abragam's group have also observed the Overhauser effect in liquids containing paramagnetic impurities which has the peculiar property that there is at first a dim. inution in the nuclear signal (Underhauser effect). The increase in intensity obtainable could be invaluable in the study of weakly magnetic nuclei. J. G. Powles (London) discussed measurements on polyisobutylene solutions in organic liquids over a wide range of concentration which give information on chain motion in this polymer. J. Hue and J. Seiden (Paris) have produced a simplified theory of double resonance.

A study of hyperconjugation in molecules by means of microwave spectroscopy was reported by J. Sheridan et al. (Birmingham). Results by the same method on formic acid were given by R. Wertheimer (Paris). J. Bonanomi et al. (Neuchâtel) have constructed an ammonia 'maser' and have studied particularly the reproducibility of such equipment. This communication provoked the usual argument about the meaning of time. Prof. A. Kastler (Paris) gave a review of his work on magnetic resonance in optically excited systems of various types.

The last session was concerned with nuclear quadrupole resonance. Prof. Kastler pointed out that Maupertuis, a native of St. Malo, was the first to measure the departure of the earth from sphericity and hence was one of the first to measure a quadrupole moment. F. Lurcat (Paris) showed how one can obtain macroscopic equations of motion for quadrupole resonance absorption corresponding to the Bloch equations for nuclear resonance. M. Buyle-Bodin (Grenoble) has studied the phase transformations in $p$-dichlorobenzene by means of quadrupole resonance.

The Conference was organized by Prof. R. Freymann (Rennes) and Dr. J. Le Bot (St. Malo-Rennes), and was supported financially by French industry. The conference was made royally welcome by the Municipality of St. Malo. The publication of the report and preparation of preprints were undertaken again by Dr. G. J. Béné and the Geneva group.

The Colloque A.M.P.E.R.E. 1958 will be held in Paris during June. Information concerning the Colloque A.M.P.E.R.E. can be obtained from Prof. R. Freymann, University of Rennes, or from Dr. J. G. Powles, Physics Department, Queen Mary College, London, E.1.
J. G. Powles

\section{THE THIRD LAW OF THERMODYNAMICS}

$T$ THE "Year Book of the Physical Society, 1956", the second in the series, contains the texts of the fortieth Guthrie Lecture and the eleventh Holweck Lecture; the addresses delivered by the recipients of the thirty-second Duddell Medal and the eleventh Charles Vernon Boys Prize; abstracts of the discourses given at the fortieth Physical Society exhibition of scientific instruments and apparatus ; obituary notices of fourteen fellows including Irene JoliotCurie, A. O. Rankine, H. R. Robinson, S. Whitehead and R. W. Wood ; and the report of the Council of the Society for 1955 .

In his Guthrie Lecture which he delivered on March 13, 1956, the late Sir Francis Simon gives a historical and critical survey of the third law of thermodynamics. In 1906, in a paper entitled "On the Calculation of Chemical Equilibria from Thermal Measurements", W. Nernst enunciated his so-called Heat Theorem which has now become the third law, and although at first it was considered mainly as a means of predicting chemical equilibria and directly applicable only to condensed systems, its later formulation as the law of the unattainability of absolute zero, and its extension to gaseous systems, led to Nernst's claim that it was a law of general validity. However, subsequent investigations diselosed a number of notable discrepancies, and the law was rejected by many physicists. In 1927 , Simon gave a new formulation of the law, which states that the entropy of all factors within a system that are in internal thermodynamic equilibrium disappears at absolute zero, and in this form the law covers all cases without exception. Sir Francis mentions that he was first a pupil, then a research assistant and finally a colleague of Nernst, and was thus close enough to obtain a very clear picture of what Nernst had in mind, but yet not so close as to be completely dominated by Nernst. In fact, some of the strongest opposition to Simon's new formulation of the third law came from Nernst himself. Turning to recent experimental developments, Sir Francis deals with spin systems, and indicates the various sub-systems which must exist within a substance, some of which are in internal equilibrium and lose their entropy at some low temperature, and others in which the disorder persists down to the lowest temperatures. Finally, he poses the question as to the present usefulness of the third law, and maintains that it has outlived most of its usefulness in the field for which it was originally intended, namely, chemical gas reactions, since the corresponding condensed phases may not be in internal thermodynamic equilibrium. Absolute zero of temperature is no longer a point of reference for the thermodynamic calculations in these cases, and we must take the quantum statistical expression for the entropy of the gas to find out whether the condensed phase at absolute zero is in perfect order or whether it has a frozen-in disorder. In low-temperature physics, however, the third law is a most important guide since most of the systems of interest to physicists are not liable to become frozen in. Moreover, those cases where apparent discrepancies with the third law have been discovered have led to a better understanding of many properties of the condensed phase.

The Holweck Lecture, entitled "The Diffusion of Visible Light by Cubic Crystals", was delivered by 
Prof. J.-P. Mathieu. He descrites how the diffusion, which is anisotropic even in cubic crystals, enables one to find the modes to which every observed line of the vibration spectrum belongs, and outlines some applications of the method to the study of crystal structure and the nature of order-disorder transformations. The early history of the travelling wave tube was the subject of the address given by $R$. Kompfner, the Duddell Medallist, and crystals of silver halides, by J. W. Mitchell, the Vernon Boys Prizeman.

S. Weintroub

\section{POLYESTER WAX}

\section{A NEW RIBBONING EMBEDDING MEDIUM FOR HISTOLOGY}

\section{By DRo H. F. STEEDMAN}

\section{Department of Zoology, University of Glasgow}

$\mathbf{I}_{\mathrm{r}}^{\mathrm{N}}$

VESTIGATION of synthetic waxes during the past ten years indicates that there are some which possess advantages over paraffin wax for histological purposes. Of more than fifty which were subjected to detailed examination 400 polyethylene glycol distearate (Anchor Chemical Co., Ltd., Manchester 11) proved to be the most suitable. To this was added cetyl alcohol as an extending agent, and the mixture is referred to as polyester wax. The amounts used are 400 polyethylene glycol distearate $99 \mathrm{gm}$., cetyl alcohol $1 \mathrm{gm}$.

This mixture has the following features :

(1) Melting point $37^{\circ} \mathrm{C}$.

(2) Insoluble in cold water.

(3) Soluble in alcohols, ethers, esters, ketones, hydrocarbons, etc.

(4) Almost opaque.

(5) Circular, notched crystals.

(6) Easy sectioning, ribboning, and flattening of sections and bbons.

(7) No electrifleation of ribbons during cutting.

(8) Good water tolerance.

(9) Flattening of sections or ribbons on solutions of stains such as methylene blue permits staining of sections through the wax, as with ester wax (ref. 1)

(10) Sections of 2 microns and more may be cut at a room temperature between $10^{\circ}$ and $22^{\circ} \mathrm{C}$.

The main advantage which this wax possesses over paraffin wax and ester wax ${ }^{\mathbf{1 , 2}}$ is its low melting point. This reduces hardening and shrinkage of tissues, as well as other heat-induced artefacts.

Associated with the use of low-temperature infiltration waxes are the two major problems of complete removal of the clearing agent or wax solvent from the specimen during its infiltration with wax, and of adhesion of tissue sections to microscope slides.

Infiltration. During infiltration it is essential that the wax solvent should be removed entirely from the specimen, and this usually takes place in two waysby diffusion into the wax, and by evaporation. At $52^{\circ}$ C. when xylene is used, as with paraffin wax, both processes operate simultaneously. When a non. volatile wax solvent is used, such as cedarwood oil, removal takes place by diffusion only. Such a process is slow and necessitates the use of several changes of wax.

When the melting point of the wax is as low as $37^{\circ} \mathrm{C}$. both processes are greatly reduced in speed, because the decreased temperature leads to increased viscosity of the wax solvent or clearing agent, and hence to slower diffusion from the specimen. Heavy, non-volatile solvents such as cedarwood oil are therefore not recommended for use with polyester wax, unless mechanical stirring is employed during infiltration. Alcohols, ethers, and esters are recommended, or hydrocarbons such as xylene or ligroin for those who prefer them. A solution of 96 per cent ethyl alcohol and 4 per cent water is excellent.

Adhesion of sections. 'Tissues infiltrated with wax at $37^{\circ} \mathrm{C}$. are not heat-denatured to the same extent as those infiltrated at $52^{\circ} \mathrm{C}$. or above. Tissue sections cut in this wax must also be dried at low temperatures-18-25 $5^{\circ}$ C. As a consequence of this, sections cut in polyester wax, when taken down to water for staining, tend to wash off the microscope slide very easily, because the section proteins absorb water more readily, and because adhesives such as egg albumen have not been baked into a condition of relative hardness and insolubility, as is the case when they are dried at $52^{\circ} \mathrm{C}$. All the known adhesives for tissue sections failed to prevent the loosening of polyester wax sections, and more than eighty new reagents for this purpose were investigated. Of these, amylo-pectin (Messrs. Brown and Polson, Ltd., Industrial Products Division, Trafford Park, Manchester 17) proved to be the most successful. It will stick sections to glass more firmly than any other medium, but when dissolved in water it breaks down and loses its adhesive properties after eight days. A preservative is therefore employed. The solution is made up as follows :

\section{Amylopectin solution:}

(1) Boil a litre of distilled water and add to it while still boiling Trading Estate, Pontypridd, Glam. (Nipa Laboratories, Ltd., Treforest (2) Cool $200 \mathrm{ml}$. of this solution. Stir until the ester is dissolved. $2 \mathrm{gm}$. of amylopectin. This will not dissolve but will lie as a white powder at the bottom of the beaker.

(3) Raise the $800 \mathrm{ml}$. to the boil and add slowly, with stirring, the $200 \mathrm{ml}$. containing the amylopectin. A bluish, opalescent sus: Always shake it before use, and do not filter.

The complete method of using polyester wax is as follows : (1) Bring fixed material to 96 per cent ethyl alcohol, 4 per cent
water; or bring to absolute alcohol, 'Cellosolve', or xylene as desired. (2) Transfer the tissue either to a mixture of any of the above fluids and wax, or directly to pure wax. The inflitration time is proximately equal to that when using paraffin wax, plus one-third. (3) Make a block as with paraffin wax. Do not immerse the molten wax in water, but leave to cool without further attention. It is not (4) Cut sections at the block with cold water.

(4) Cut sections at the speed of paraffin wax sectioning-approx(5) $80-100$ per min. Do not handle the block.

(5) Float the sections or ribbons on to the amylopectin solution
(ven above, either on a microscope slide or in (B) Flatten the on a microscope slide or in a dish (6) Flatten the sections by leaving them on the amylopectin process for 5-10 min. at a room temperature of $18^{\circ} \mathrm{C}$., or speed this plate at $25^{\circ}$ C. for about 30 sec.

(7) Drain the excess amylopectin solution off the slide and dry the sections overnight at room temperature or at about $25^{\circ} \mathrm{C}$. As excess amylopectin will show as fine black dots under the microscope, the slide must be well drained before drying.

(8) From then on treat as paraffin wax seotions.

Polyester wax tends to become softer after it has been continuously heated in an oven at $37^{\circ} \mathrm{C}$. for several weeks.

It is considered that this wax could take the place of paraffin wax both for routine purposes and for research work. Its low melting point permits its use without expensive infiltration ovens, and for this reason histological practice might be brought within the reach of schools and colleges where it had not been possible previously for financial reasons.

It is hoped to publish a more detailed account elsewhere.

'Steedman, H. F., Quart. J. Micro. Sci., 88, 123 (1947).

${ }^{2}$ Chesterman, W., and Leach, E. H., Quart. J. Micro. Sci., 97, 593 (1956). 